\title{
Aspect Orientation for Composite Services in the Telecommunication Domain
}

\author{
Jörg Niemöller, Roman Levenshteyn, Eugen Freiter, Konstantinos Vandikas, \\ Raphaël Quinet, and Ioannis Fikouras
}

\author{
Ericsson $\mathrm{GmbH}$ \\ Ericsson Allee 1, 52134 Herzogenrath, Germany \\ \{joerg.niemoeller, roman. levensteyn\} @ericsson.com, \\ \{eugen.freiter, konstantinos.vandikas\} @ericsson.com, \\ \{raphael.quinet, ioannis.fikouras\}@ericsson.com
}

\begin{abstract}
Telecommunication network operators have specific requirements on services offered through their network, which are frequently independent of the core business logic of the service. As an example, these requirements ensure monitoring of user activities for charging purposes or allow controlling parameters that influence the quality of service. In order to satisfy these demands, services are typically tailor-made to support these supplementary features next to their core business logic. As a result, their implementation becomes tangled and specialized. This is identified as a major obstacle for efficient service composition, because more specialized services are less suitable for being reused in different contexts. This paper describes an approach to introduce concepts of aspect-oriented programming to service composition in order to keep the implementations of telecommunication-specific requirements separated from the core business function of a service.
\end{abstract}

Keywords: AOP, IMS, Service Composition, Telecommunication.

\section{Introduction}

Telecommunication network operators aim for a service infrastructure that allows a converged usage of heterogeneous services. They rely on cost-efficient and rapid design of new applications by re-use of already existing services. Another goal is differentiation form the competition by integrating telecommunication services with popular community services from the internet, because these services provide users with a new style of communication and social interaction. Service composition is a key technology for reaching the desired convergence within a heterogeneous service environment. This paper is based on an approach for service composition [1] that supports multiple service technologies to be used within a single composite service.

Typically, operators from the telecommunication domain require support for specific supplementary functions from all services provided through their network. These functions, for example, allow charging for service usage, collect statistics or help controlling the service quality. 
This paper analyzes the design of converged composite services that need to consider specific requirements of the telecommunication domain. Furthermore, this paper outlines that these requirements often imply cross-cutting implementations, therefore they are a severe obstacle for efficient composition of services. A solution is presented that combines data-driven composition of heterogeneous services with techniques from aspect oriented programming (AOP). This includes weaving based on the unique elements of the composition environment. The paper explores a solution in which aspects are modeled as services and selected through constraint-based service selection, thus concepts known from the underlying service composition approach are applied to AOP.

\section{Related Work}

The central problem of composing applications according to cross-cutting concerns is motivated in [2]. That paper defines common concepts and terminology of the AOP domain like cross-cutting concerns, aspects, point-cut, advice, and weaving. In our paper we follow this terminology.

AspectJ [3] was one of the first implementations of the AOP paradigms as proposed in [2]. AspectJ extends the Java language by new elements that allow the definition of point-cuts and advice code. Weaving is performed prior to execution, resulting in a regular Java application including injected advice code.

JBOSS-AOP [4] introduces weaving on byte-code level rather than on source code. This allows online weaving at runtime rather than offline weaving that is performed prior to runtime, like for example in AspectJ. In this paper we apply online weaving, since the dynamic run-time behavior of composite services means that information for effective identification of relevant join-points is not available at design time.

AO4BPEL [5] is an aspect-oriented extension for business process execution language (BPEL) [6], [7]. Online weaving is used in order to dynamically add or remove aspects from a BPEL process. Each activity within a BPEL workflow process can serve as join-point. Point-cuts can span over multiple BPEL processes and attributes of the BPEL processes can be used in order to identify relevant join-points.

A framework that uses aspect orientation for dynamic selection of web services is presented in [9]. The goal here is to dynamically select the web services to be used within client-applications. An intermediate layer for managing web services and decoupling them from applications is introduced based on AOP principles. Aspects are used in order to flexibly redirect web service invocations to alternative services, thus flexibly binding web services to clients.

This paper is based on the service composition technology described in [1]. According to this approach [1], an abstract description of constituent services function and capabilities is the base of composition. Service templates are used to describe which constituent services shall be used as components of the composition. This is achieved by using constraints for specifying the properties a service required to be selected for execution. The constituent services to be used as components of a composite application are therefore selected according to their abstract description, rather than pointing directly to a concrete service. A service skeleton represents a model of a 
composite service. It combines service templates with control elements that steer the composition process.

In order to execute the composite service, the skeleton is executed step-by-step by a composition engine. In this process, constituent services are selected by satisfying the constraints specified by a service template. These constraints perform a selection among the descriptions of all available services, stored in a service repository. The service descriptions contain abstract descriptions of the service function and capabilities, coupled with binding information. For example, a service that provides the geographical location of a subscriber might be described by its function "positioning" and by its capability to provide the position with certain accuracy. The composition is session based and all services within a composition session have access to a shared state, which can be used for data exchange between services.

\section{Crosscutting in the Telecommunication Domain}

The requirements of a telecommunication network operator are mainly driven by business models, legal requirements and standardization. Besides the functionality offered as end-user services to their subscribers, multiple supplementary functions are usually required. Examples include the collection of information for charging and billing purposes, or monitoring and control of service quality. Support for this kind of functionality is usually an integral part of a service that targets a telecommunication network, but it considerably increases the costs for service design and modification. Thus, methods reducing the investments into system integration, customization and new design are of particular importance for operators.

Furthermore, services from third-party providers often do not meet the very specific requirements of a network operator. Customization of these services is usually not feasible and not desired as this would create very specialized services that are not suitable for broad re-use in new contexts.

Service composition allows the creation of new applications by re-using existing constituent services. A composition technology like the one described in [1] provides simple tools that allow the creation and modification of composite applications in a very cost efficient way. Fig. 1 shows a simplified skeleton of a composite service that provides a weather forecast for the user's geographical location. It combines services that retrieve the user position with a weather forecast service. Depending on the user preferences, further services might generate a map illustrating the forecast and finally send an SMS or MMS to the user.

When offering this service through a telecommunication network, the operator usually requires support for charging. In addition, logging of all used services for statistical purposes might be required as well as setting and monitoring of service quality parameters. These supplementary features do not provide a service directly to the end-user, but they help the operator controlling service and network operation. For this reason they are often referred to as non-functional requirements.

Using the design methods of service composition, the supplementary functions could be implemented by selecting only those constituent services that already contain support for such functions. Alternatively, additional services can be included into the 
composition, which contribute only the supplementary features to the overall composite service. The resulting composite service is depicted in Fig. 1 on the right side.

One observation is that additional services providing supplementary functions spread across the whole composition. They cannot be implemented within a single, well encapsulated entity due to their inherent need to interact with the constituent services throughout the whole composite application. These supplementary functions show a property that is referred to as cross-cutting in AOP terminology. Cross-cutting caused by supplementary functions is typical for telecommunication services. Although it is not further investigated within the scope of this paper, a similar observation can be made for the domain of enterprise applications.

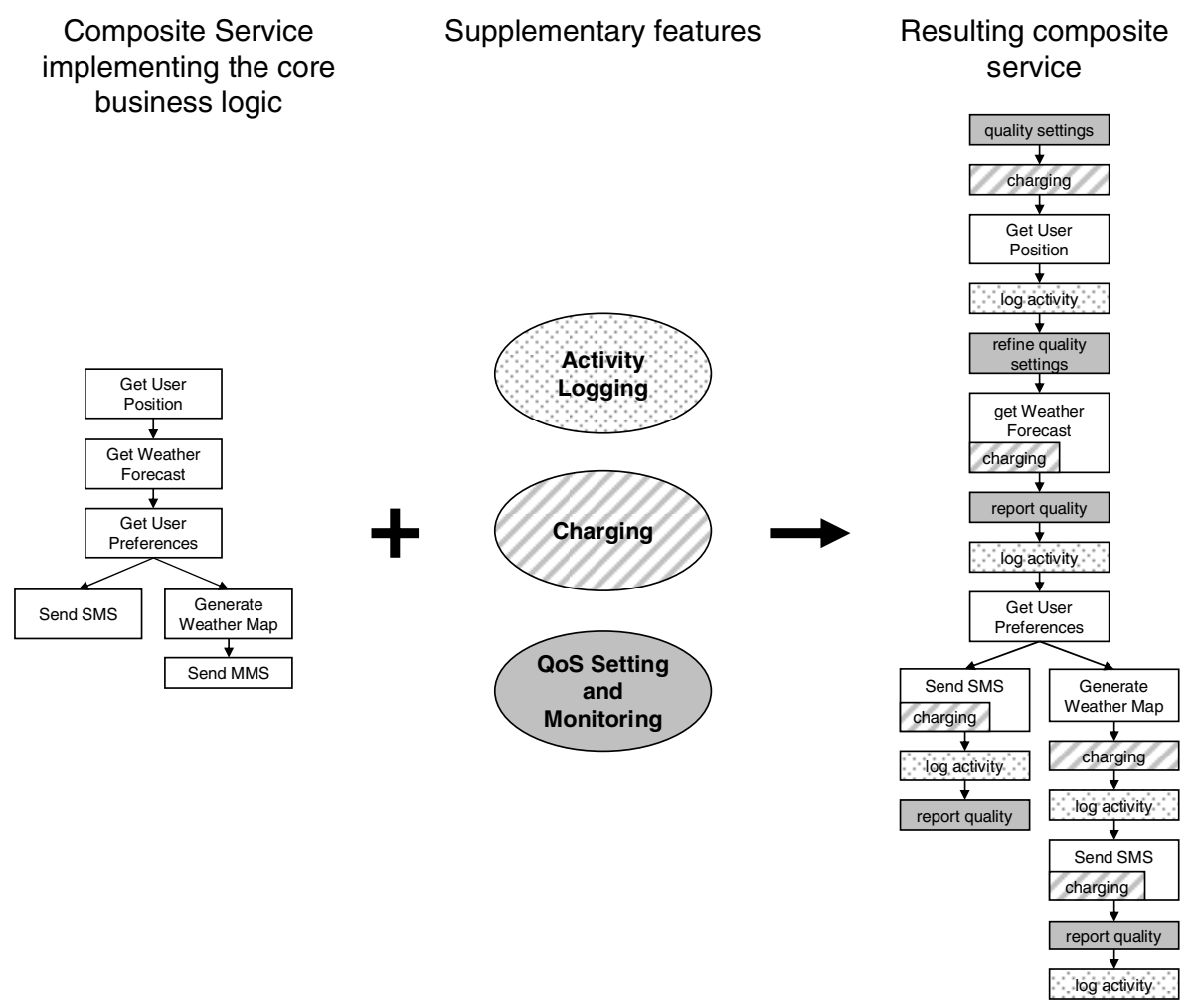

Fig. 1. Adding supplementary features to a composite service

The described method for service composition [1] is based on modules that are products of functional decomposition of an intended application. In this respect, it is not different than procedural or object oriented programming languages and equally affected from cross-cutting as described in [2]. Its advanced features like constraintbased service selection and service modeling based on abstract service descriptions are designed to handle services that are strongly encapsulated functional entities. 
Therefore, they are weakly entangled with other services. As a result, the features of service composition do not help handling cross-cutting.

Another observation is that certain services have already included the support for supplementary features. In Fig. 1, for example, the support for charging was implemented into the service "get Weather Forecast". A service like this is multifunctional, which increases its specialization. It is tailor-made for certain scenarios and might not fit in other scenarios. Some of the multiple functions it provides might interfere with other services or it would need to operate differently. For example, the charging enhanced weather forecast service cannot be used if a different charging method needs to be applied although its basic function alone, the weather forecast, might fit.

Composite services can be created with comparably small investments into service design together with short lead-times. This cost efficiency of composite services depends to a great extent on the availability of constituent services that can be flexibly re-used in different contexts. Such a service is virtually lean and functionally pure, thus it focuses on its single main function. The more specialized a service is, the less suitable it becomes for broad use within composition.

Aspect orientation of the composition environment reduces the need for implementing supplementary functions into constituent services or into composition skeletons. They can be implemented separately and applied if needed. The following chapter outlines an AOP enhanced composition environment.

\section{AOP for Composite Services}

Aspect orientation is proposed to become an integral part of the composition engine that executes a composite service by interpreting the respective service skeletons. This integration is practical as most join-points lie within the execution steps of the composite service. In order to catch relevant join-points, a weaving function allocated within the composition engine monitors the skeleton execution and applies advice when necessary. This process is steered by point-cut definition expressed within a weaving language that is tailor-made for the underlying service composition technology.

Execution of composite services as described in [1] is based on service selection at runtime leading to late-binding of constituent services. Furthermore, the requirements and conditions for service selection, which are expressed in terms of constraints, are dynamic. They might, for example, depend on services that were executed previously within the same composition session. As this dynamic service selection is the central process of the described service composition technology, many useful join-points originate within this service selection process and are in turn volatile and dynamic. Thus, weaving prior to the execution of a composite service is in many cases not possible. For this reason an event driven online weaving approach was chosen.

\subsection{Weaving Definition and Join-Points}

Weaving refers to composing an application from a target application where an additional function and aspects are desired that implement this function. The base and target of the proposed aspect enabled environment is the step-by-step execution of 
composite service skeletons. At each step of the execution where a potential joinpoint resides, point-cut definitions are interpreted in order to identify and execute those point-cuts that apply here. If advice is defined for the currently reached pointcut, it is executed. The elements of the skeleton and therefore the steps in the execution of a composite service represent the source for join-points and the target for point-cuts and advices. Basic join-points in this environment are for example:

- The start and end of a skeleton execution. This join-point would allow to execute an advice prior to or after the execution of the actual composite service.

- The start of a service selection. This join-point would allow to analyze the starting of service selection and to influence it by e.g. the addition of constraints.

- A list of services that satisfy the constraints was returned from the service repository. This join-point occurs as one step within the constituent service selection. A list of all services that qualify for being selected according to constraints is available. This join-point is helpful if, for example, advice needs to further prioritize certain services.

- A service is invoked. This join-point marks the invocation of a selected service. Advice can be used here for example to influence the parameters that are used in the service call.

- A return value is received. This join-point marks the return of a result from an invoked service. Here an evaluation of the result is possible before it is processed by the target composite service.

- A message is received. This join-point marks the reception of a message from an external protocol in the composition engine, for example a SIP message.

Some of the aforementioned basic join-points correspond to the elements of the skeleton language like, for example, the start element or the service template, and some originate in the end-to-end communication session, like the SIP message reception. Furthermore, events from the composition environment and events in the composite service execution are reflected uniformly within the weaving language by considering that reaching a new execution step and sub-step is an event in itself.

It is important to note that weaving instructions are stored and managed separately from composite services. This way, they can be applied and modified separately, without opening the target service. Thus, aspects can be added or removed independently of composite service design.

The weaving language is kept simple and intuitive and it allows the definition of point-cuts by means of weaving instructions. These weaving instructions consist of condition, control instructions and advice.

IF (<condition>) DO <control instructions > <advice>

Reaching a potential join-point in the skeleton execution triggers an event. If such an event occurs, the applied weaving instructions are evaluated. At this point it is important to highlight that multiple, independently specified sets of weaving instructions might be applicable. All of them are considered.

The keyword "IF" marks the start of a weaving instruction and precedes the condition element. The purpose of the condition element is to verify if this particular weaving instruction matches the currently considered event representing a join-point. 
Furthermore, it specifies if and which advice shall be executed. A central element here is the join-point or event type that triggers the weaving. Additionally, further conditions might exist, that evaluate variables stored in the shared state of the composition session. Depending on the event type, further data might be subject to conditions. For example, for join-points that reside within the context of service selection, the condition might be defined based on currently used selection constraints. If a condition cannot be evaluated because data is not available in the current context, this particular weaving instruction is ignored and weaving proceeds.

If the condition is found to be true, the respective advice is executed. The definition of advice and its mode of execution are specified after the keyword "DO" within a weaving instruction.

It is important to note that some combinations of join-points and additional conditions might fail. For example a condition based on constituent service selection combined with the event for skeleton start might never be evaluated because the required information is not available in this context. This weaving instruction will never apply.

By means of the optional control instruction, the execution of the advice code can be steered. By default the advice code is executed synchronously by halting the skeleton execution at the join-point. Skeleton execution resumes after the advice execution has been finished. By means of the control instruction, asynchronous advice execution can be selected (keyword "ASYNC"). Furthermore the advice execution can be delayed after the action in the skeleton execution that is marked by the join-point (Keyword "AFTER"). With this behavior, the start of the advice execution can for example wait for results of the action that is connected to the join-point. The default is to execute advice immediately at reaching the join-point.

\subsection{Advice Selection and Execution}

The presented approach allows different ways to implement advice:

- As separate composition skeleton

- As inline command within the weaving instruction

- As external service

If advice is implemented as separate skeleton, the weaving instruction explicitly refers to this skeleton. In the following example the join-point is the execution of a service template (event SERVICESELECT).

IF (event=SERVICESELECT, constraint="srv=user_profile")

DO AFTER SKELETON(alt_user_profile)

Here, the weaving only applies if this service template contains a selection constraint requiring a service that is described as "user_profile". This service, for example, loads a profile of the user from an external database into the shared state of the composition session. The weaving instruction specifies that once the service template is finished, thus after the selected service was executed, a skeleton called "alt_user_profile" is started as advice. This advice skeleton might, for example, contain functionality that processes and modifies user profile information. The composition engine continues execution within the advice skeleton. If the advice skeleton is finished, the execution 
resumes in the original skeleton. Being implemented as skeleton, advice can in turn be subject to further weaving when being executed.

Another possibility to implement advice would be an inline command given from within the weaving instruction. This way, simple operations like a change within a shared state variable can be initiated without the overhead of an external implementation of such a basic operation. The following example shows the usage of commands as advice:

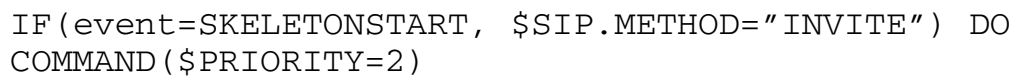

At skeleton start and if a SIP INVITE message was received according to the value of a shared state variable \$SIP.METHOD, the command sets the shared state variable \$PRIORITY to the value 2 .

The most flexible possibility to implement advice would be to implement it as an external service, for example as a web service. This separates the advice implementation from the implementation of the targeted composite services. Services are self contained entities with strongly encapsulated functions that interact through well defined APIs and protocols. Using an external service as advice implies the invocation of this advice service at join-points. Thus, a service invocation is directly weaved into the target application rather than the advice itself, which stays a separate process.

In the simplest cases, an external service could be used as advice by directly and statically addressing it from the weaving instruction.

Tooling of the underlying service composition environment provides an even more dynamic and flexible method.. The model-driven composition approach and in particular the data and constraint based selection of services may be applied to the selection of services that implement an aspect. For this purpose, the weaving instruction specifies selection constraints rather than pointing statically to an advice. Like any other service in the composition environment, services that implement advices are formally described within a service repository. Thus, aspects are implemented and exposed in the same way as the constituent services that are used in skeleton based composition.

The service description contains binding information and abstract description of the service function and capabilities. Being based on constraints, advice selection in the weaving process is based on abstract properties. The same constraint expressions that are used in skeleton controlled composition apply in weaving instructions.

The following example of a weaving instruction adds functionality to count the number of skeleton starts within the environment to composite services.

\section{IF (event=SKELETONSTART) DO SELECT (srv="skeleton_count")}

The keyword "SELECT" instructs to use the following parameters as constraints for service selection in order to find the advice to be executed. In this example, the constraint demands to use a service that is described by the property "srv" as "skeleton_count". This constraint is matched against the service repository in order to find applicable advice. The aspect is applied by executing the selected advice service.

In this example, the same function could be added to a skeleton by inserting an additional service template directly after the skeleton start element. Even the same constraint for service selection can be used, potentially finding and invoking the same 
service. This means that a service could be used either as a constituent service within a composition or the same service could serve as advice within aspect weaving.

The skeleton counting example above describes a typical supplementary function within a network. Its conventional implementation with additional service templates would cross-cut throughout all composite services. By means of the weaving instructions, an alternative mechanism is available that complements the composition without changing in the composition skeleton. In this respect, it is important to note that both the skeleton based composition and the weaving are based on the same enablers like constraint based service selection, abstract description of the services and the shared state of the composition session.

The underlying composition environment supports services from various service technology worlds. Currently, next to SIP and Web Services, AJAX services can be used and Enterprise Service Buses (ESBs) are supported. The composition core process of constraint based service selection is agnostic of the technology of constituent services due to using abstract description of a service's functional properties rather than the technological details of its implementation. For the application of aspects this means in principle, advice can be implemented based on any service technology that is supported by the composition environment. In practice, some service technologies like IMS/SIP, where services are persistent within end-to-end user communication sessions, is less suitable for advice implementation. Instead, aspects can be implemented using technologies like web services or AJAX, which are based on requestresponse usage schemes.

\subsection{Data Exchange with Advice Services}

An important issue is how access to data is granted to the advice. There are two philosophies for data handling: the full direct access to all data from the advice code or the encapsulation of the advice in a way that allows data exchange with the target application only through dedicated APIs. The presented approach uses both methods depending on the advice implementation.

If an aspect is implemented as separate skeleton, this skeleton would be executed as integral part of the target skeleton and within the same composition session. This implies full access to all run-time data of the composition.

If an aspect is implemented as separate external service, it is encapsulated and needs to exchange data through dedicated APIs. For this purpose, two possibilities are available:

- Data exchange through an API exposed by the composition environment.

- Data exchange defined in weaving instruction using the service API.

For the first alternative, access to shared state is provided through an external API of the composition engine. This API allows reading and writing shared state variables. This method requires the advice service to use this particular API.

The latter alternative takes into account that advice invocations are service calls, which can be parameterized and which might provide a return value. The service parameters to be applied to the advice service invocation are defined in the weaving instruction. The values used in parameters can for example originate in shared state variables within the composition session. Furthermore, the return-value of the advice 
service can be connected with a shared state variable. The following example shows the concept:

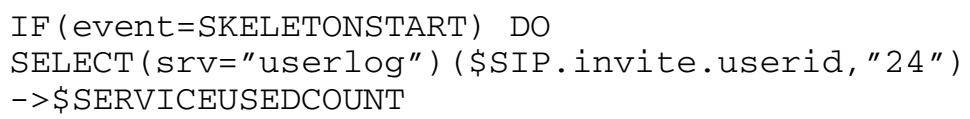

Here, at every start of a skeleton, a service shall be called, that logs the skeleton usage per user. The user address, as received from SIP, was stored within a shared state variable and it is used here as first parameter. The second parameter is a constant that specifies the time interval for logging. Here 24 hours is used. As return value, the service provides the number of skeleton invocations by this user within the specified time interval. This return value is directed into the shared-state variable \$SERVICEUSEDCOUNT and available for further processing within the composition session. This processing might be implemented e.g. by further aspects applied to this composition. As shown above, shared state can be used by aspects for exchanging data between each other. Furthermore, shared state can be used to share data with the target application. The target application is not aware of the presence of aspects but it considers the variables that are used by the aspects as part of the run-time environment.

Data exchange through the weaving instruction and the API of the advice service allows implementing aspects without considering additional APIs that are specific to the composition environment. The resulting services are more generic, thus more suitable to be used in different contexts.

\section{Example}

This example demonstrates the addition of functionality that logs constituent service usage to a composite service. As a base, the service that was already outlined in Fig. 1 is used. It provides an automatically localized weather forecast service. Additionally, the service takes into account user preferences regarding the delivery of results.

Fig. 2 shows the composition skeleton that implements this service. The white boxes in the skeleton are service templates, which imply constraint based selection of services. They are complemented by structural elements that mark the start and end of the skeleton and provide conditional branching of the execution.

The function to be added is logging of constituent service usage. Implementing this within the skeleton would mean adding an additional service template after each already existing service template. This additional service is shown on the right side in Fig. 2. It shows the six locations in the skeleton where this service template would need to be added.

The same result can be achieved by means of weaving without changing the original skeleton. The following weaving instruction inserts the logging service at all six join-points:

IF (event=SERVICESLECT) DO AFTER

SELECT ( srV="logging") (\$USERID, \$LASTCONSTRAINT.SRV)

$->$ \$NUMBER 


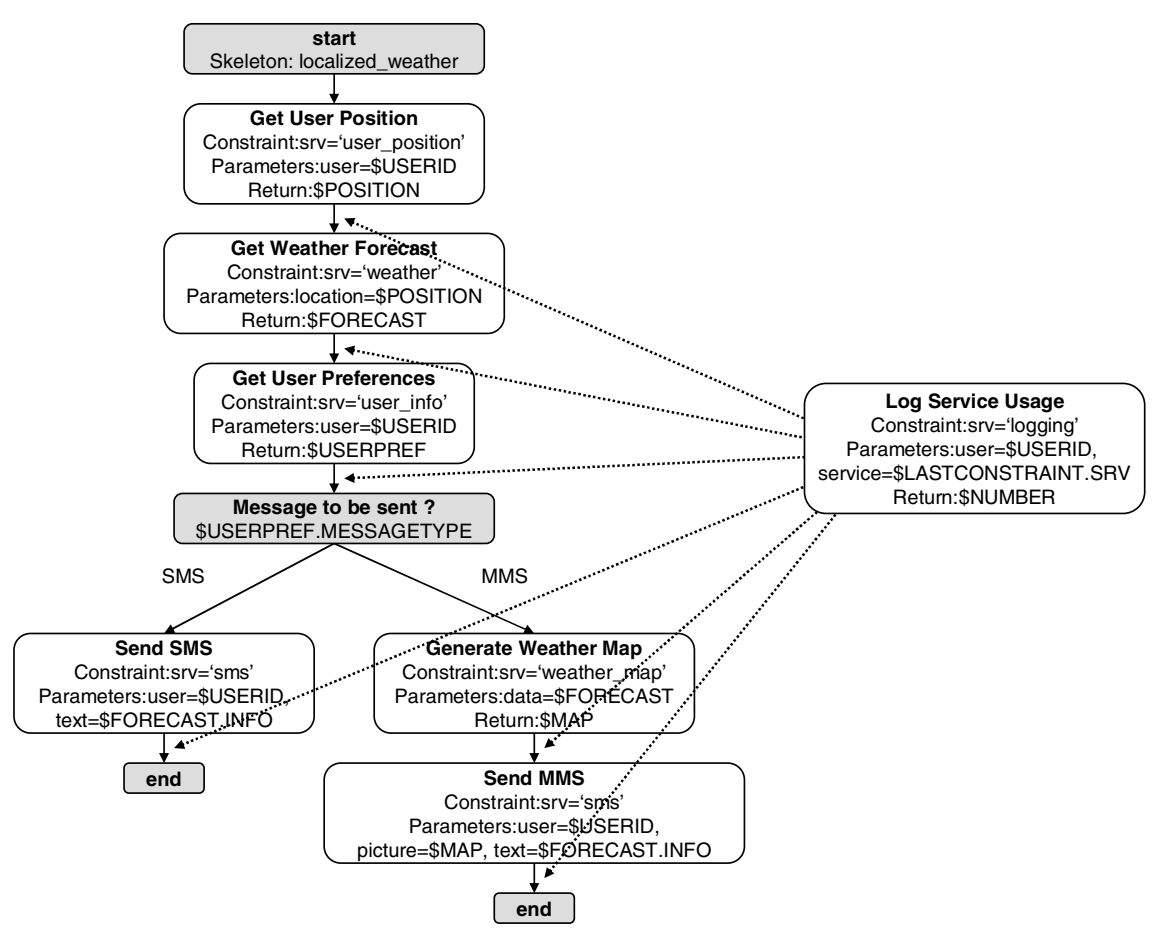

Fig. 2. Example skeleton implementing location based weather forecast service

The aspect shall be applied after each service selection. The respective join-points correspond to the event SERVICESELECT. The keyword 'AFTER' instructs to wait with the advice execution until the execution of the skeleton selected service was finished.

This example shows the similarities in the skeleton controlled composition and weaving of aspects. The selection of the additional logging service is based on the same constraint regardless if it is applied through additional service templates added to the skeleton or by means of weaving. The parameters for service invocation and the return value are connected to the same shared state variables. Nevertheless, the aspect oriented approach implies two important advantages. It does not modify the original service skeleton and it can easily be applied to multiple composite services at once. It therefore helps to keep the service lean and it allows a broad deployment and distribution of additional functionality within a service domain.

\section{Summary and Discussion of the Approach}

The presented approach uses dynamic weaving at run-time, based on intercepting events, which reflect the process of composition execution or originate in the run-time 
environment. Weaving is applied conditionally, depending on the state of the composite service execution. Selection of advice is dynamic and steered by constraints. Furthermore, the addition of aspects is decoupled from the design of a composite service. As a result, aspects not only improve the modularity, but allow integration of an existing service into a network environment by adding specific functions that are required within this network domain.

In the presented approach, an aspect can be any service that is formally described and exposed within the composition environment through a service repository. In principle, this means that the same service can be used as constituent service in skeleton based composition as well as being applied as aspect through weaving. In either way, it contributes to the overall composition of an application. Weaving is in this respect an additional composition mechanism that complements the skeleton based approach. In this sense, point-cuts, as expressed in the weaving instructions, can be considered to constitute a new type of structural constraint for the overall composition that comprises skeleton execution and weaving.

The skeleton provides a model of the composite service by means of constraints in the same way as the weaving instructions, being based on constraints, provide an aspect model. Thus, not only skeleton based service composition was enhanced by concepts of AOP. By adding aspect orientation to the composition environment as presented in this paper, also aspect weaving was enhanced by techniques that originate in data-driven service composition. This implies that characteristics of the composition environment, like late-binding and loose-coupling of constituent services, can be applied to aspects. The just-in-time selection of aspects results in late-binding. The selection being based on abstract constraints means loose coupling of aspects.

In the presented approach, activities like constraint based service selection and service invocation are treated as join-points regardless if they occur in the execution of skeletons or weaving instructions. This implies that weaving execution might in turn be subject to further weaving. Furthermore, a service being invoked as advice does not differ from a service being invoked from skeleton execution. Thus, the activities of an aspect service might be subject to further weaving.

Being implemented and exposed as services, aspects, as described in this paper, are strongly encapsulated entities in the sense of SOA (Service Oriented Architectures). They offer their function through clearly defined APIs, but they are in principle not dedicated to a certain application use-case scenario. The application of aspects is kept separate and it is entirely done within the weaving instruction. As a result, aspects are implemented in a generic way considering as less information about a specific application scenario as possible. Thus they are re-useable in many contexts. This is in contrast to aspects known from other AOP approaches like AspectJ and JBOSS-AOP, where an aspect itself often contains information about where and under which conditions it can be applied.

In many scenarios, AOP is used by a single developer who implements the target application along with the implementation and application of aspects. The presented approach additionally allows using AOP for target applications that might be provided by a different administrative domain, for example, a $3^{\text {rd }}$ party service provider. In this context, the described aspect orientation concepts can be used as tools for system integration. 
The composition engine is based on a separation of service selection and service execution. Thereby, it becomes service technology agnostic to a great extent. The described weaving approach inherits this characteristic by selecting and executing advice similar to constituent services. Thus, weaving becomes as technology agnostic regarding the used aspects as the skeleton based composition is agnostic regarding constituent services. Aspects can be implemented with a different programming language or even based on a different service technology than the target application or other aspects used within the same environment. In general, aspects can be implemented based on any of the various service technologies that are supported by the composition environment. It is possible to replace an aspect by another one that is implemented using a different service technology without the need to change the composition skeleton or the weaving instructions. Both the skeleton and the weaving instructions can accommodate changes in the available services. They can be deployed in a different environment with different services and work unchanged changes as long as services that satisfy the constraints are available. In this respect, the presented approach differs from other AOP enhanced environments like, for example, AO4BPEL [5]. In AO4BPEL the composition mechanism and the aspects are mostly based on BPEL workflow processes and web service technology. Aspects are not selected as dynamically as presented here though abstract modeling based on constraints.

Furthermore, the approach presented in this paper differs from the framework that was proposed in [9]. Rather than using aspects to modify the binding of web services and client applications as proposed in [9], services are considered to be the aspects themselves. In our approach, the binding flexibility lies within the expressiveness of the weaving instructions and the constraint based selection of advice implementing services.

Regular expressions in the weaving language, as they are known for example from JBOSS-AOP [4], would provide expressiveness to the join-point selection that is useful if complex language constructs are the base for point-cuts. Within the proposed skeleton based environment such a powerful mechanism is not needed due to the limited complexity of the join-point model based on skeleton elements and events.

The presented way of online weaving implies that the selection of aspects at different join points is decoupled from each other while alternative aspects for the same functionality are available. This might for example lead to the problem that whenever a logging aspect has to be applied, a different one is selected and the complete logging becomes inconsistent. In order to address this problem, we are working on coordination in-between the aspect selections.

The described online weaving evaluates the weaving instructions at every potential join point. If extensive collections of weaving instructions are applicable, this approach might considerably impact performance of the composite service execution. In order to improve in this respect and improve run-time performance of the composite application, static offline weaving might be applied if possible. Due to the dynamic nature of the weaving concept that is characterized by weaving instructions based on run-time conditions and dynamic constraint based advice selection, a full offline weaving is usually not possible, but partial weaving might be possible offline. It can be applied as pre-processing that optimizes the overall composition process. 
In order to allow design, deployment and application of aspects in a user-friendly way, we are currently integrating the development of weaving instructions and the management of aspects into the existing development and management tools for composite services.

\section{Conclusion}

In this paper, we have identified how frequently required supplementary functions, which are essential in the telecommunication domain, affect service composition. We have outlined the cross-cutting nature of many of these supplementary functions. If these supplementary functions are implemented directly into the services, they lead to complex, multi-functional and therefore specialized services.

One major benefit promised by service composition is fast and cost-efficient design cycles enabled by re-using service components to a great extent. In order to reach this goal, service composition relies on the availability of lean constituent services. We have outlined how aspect oriented software design helps keeping services lean as it allows separating these cross-cutting supplementary functions from the core function of the service. This is achieved by the introduction of aspect weaving in the composition environment.

Furthermore, this paper has not only applied aspect oriented design principles to a new domain of composite services based on its specific composition paradigm and language. It has rather shown that aspect weaving can reside as an additional and complementary composition method besides a skeleton based approach. We have shown that aspects can be implemented as services and added to a composition following the same constraint based mechanism that is used for skeleton driven selection of constituent services. The implementation of the presented concepts is ongoing.

We have outlined that the described methodology does not only support the composite service designer, but it allows broad application and management of additional functions, e.g. applied to multiple services within a domain. Aspects can be applied to a number of applications at once and automatically. This way, services that are available from third-party providers could be adapted without changes. This feature is especially interesting for telecommunication network operators, considering their specific needs regarding supplementary functions. In this respect, the global management of weaving instructions deserves further investigation in order to control how new functions are distributed to multiple applications.

\section{References}

1. Dinsing, T., Eriksson, G., Fikouras, I., Gronowski, K., Levenshteyn, R., Pettersson, P., Wiss, P.: Service composition in IMS using Java EE SIP servlet containers. Ericsson Review 84(3), 92-96 (2007)

2. Kiczales, G., Lamping, J., Mendhekar, A., Maeda, C., Lopes, C., Loingtier, J.-M., Irwin, J.: Aspect-Oriented Programming. In: Aksit, M., Matsuoka, S. (eds.) ECOOP 1997. LNCS, vol. 1241, pp. 220-242. Springer, Heidelberg (1997) 
3. Kiczales, G., Hilsdale, E., Hugunin, J., Kersten, M., Palm, J., Griswold, W.G.: An Overview of AspectJ. In: Knudsen, J.L. (ed.) ECOOP 2001. LNCS, vol. 2072, p. 327. Springer, Heidelberg (2001)

4. Burke, B., Flury, M.: JBOSS-AOP, http: / / www . jboss.org/developers/projects/jboss/aop.jsp

5. Charfi, A., Mezini, M.: Aspect-Oriented Web Service Composition with AO4BPEL. In: Zhang, L.-J., Jeckle, M. (eds.) ECOWS 2004. LNCS, vol. 3250, pp. 168-182. Springer, Heidelberg (2004)

6. Web Services Business Process Execution Language (WSBPEL), OASIS (2007), http: / /www. oasis-open.org/committees / tc_home.php?wg_abbrev=wsbpel

7. Khalaf, R., Mukhi, N., Weerawarana, S.: Service-Oriented Composition of Web Services (WS4BPEL). In: WWW 2003 Conference, Budapest, Hungary (2003)

8. Charfi, A., Mezini, M.: AO4BPEL: An Aspect-oriented Extension to BPEL. World Wide Web Journal 10(3), 309-344 (2003)

9. Cibran, M.A., Verheecke, B., Vanderperren, W., Suvee, D., Jonkers, V.: Aspect-Oriented Programming for Dynamic Web-Service Selection. World Wide Web Journal 10(3), 212-242 (2003) 